and the BCL III Advisory Committee will be studying the updating issue in the next few years and making recommendations to Choice and ACRL for a more current review and revision cycle.

The high degree of involvement of the Choice staff and reviewer pool in the revision of BCL is indicative of their commitment to publish usable, high quality collection development resources for academic libraries.

$C \triangleleft R L$ News readers who would like to volunteer or to recommend colleagues for the librarian review stage of the BCL III project are invited to submit names and a statement of subject interest and qualifications to Virginia Clark, Editor BCL, Choice, 100 Riverview Center, Middletown, CT 06457 .

\title{
ACRL candidates, 1987 elections
}

\section{Who's who on the Spring ballot.}

he listing for each of the following candidates includes their title, institution, and institutional address.

\section{Vice-President/President-Elect}

Joseph Boissé, University Librarian, University of California, Santa Barbara, CA 93106; Joan Chambers, Director of Libraries, Colorado State University, Fort Collins, CO 80523.

\section{Board of Directors}

Director-at-Large: Charles S. Fineman, Hu manities Bibliographer, Collection Management Office, Northwestern University Library, 1935 Sheridan Road, Evanston, IL 60201-2924; Peter Malanchuk, Chairman, Department of Reference and Bibliography, 116 Library West, University of Florida, Gainesville, FL 32611.

Director-at-Large: Larry Hardesty, Director of Library Services, Eckerd College Library, P.O. Box 12560, St. Petersburg, FL 33733; Norma Yueh, Director of Library Services, Ramapo College of New Jersey, Mahwah, NJ 07430.

\section{Anthropology and Sociology Section (ANSS)}

Vice-Chair/Chair-Elect: Stephen E. MacLeod, Social Sciences Bibliographer, Green Library, Stanford University, Stanford, CA 94305.

Secretary: Deborah A. Kane, Social Science Reference Librarian, Reference Department, Univer- sity Library, University of Nebraska at Omaha, Omaha, NE 68182; Cheryl C. Kugler, Head of Monograph Services, Jean \& Alexander Heard Library, Vanderbilt University, 419 21st Ave. South, Nashville, TN 37205.

Member-at-Large: Jo Kibbee, Anthropology Subject Specialist, 100 Library, University of Illinois, Urbana, IL 61801; Virginia F. Moreland, Computer Search Coordinator, University Libraries, University of Nebraska, Lincoln, NE 68588-0410.

\section{Art Section (ARTS)}

Vice-Chair/Chair-Elect: Charles R. Smith, Humanities Reference Librarian, Sterling C. Evans Library, Texas A\&M University, College Station, TX 77843.

Secretary: Micheline Nilsen, Creative Arts Librarian, Cheever Hall, Montana State University, Bozeman, MT 59717; Stephen Allan Patrick, Head, Documents Department, Sherrod Library, East Tennessee State University, P.O. Box 22450 A, Johnson City, TN 37614-0002.

\section{Asian and African Section (AAS)}

Vice-Chair/Chair-Elect: Basima Q. Bezirgan, 5000 S. Cornell, \#7B, Chicago, IL 60615; Katharine K. Elsasser, Head, Humanities I Section, Subject Cataloging Division, Library of Congress, Washington, DC 20540.

Member-at-Large: Eleanor Murphy Daniel, 
Head, Black Studies Library, Ohio State University, 1858 Neil Avenue Mall, 210 Main Library, Columbus, OH 43210; Robert B. Marks Ridinger, Sociology/Anthropology Subject Specialist, Northern Illinois University, DeKalb, IL 60115.

\section{Bibliographic Instruction Section (BIS)}

Vice-Chair/Chair-Elect: Donald J. Kenney, Head, General Reference Department, University Libraries, Virginia Polytechnic Institute and State University, Blacksburg, VA 24061; David N. King, Graduate School of Library and Information Science, University of Illinois, 410 David Kinley Hall, 1407 W. Gregory, Urbana, IL 61801.

Secretary: Katherine Branch, Head, Access Services, Welch Medical Library, Johns Hopkins University, $1900 \mathrm{E}$. Monument St., Baltimore, MD 21205; Sharon Mader, Information Retrieval Librarian, Memphis State University Libraries, Memphis, TN 38152.

Member-at-Large: Ellen Broidy, Coordinator of Library Education Services, Main Library, University of California, Irvine, CA 92713; Ree DeDonato, Head, General Reference Center, Bobst Library, 70 Washington Square South, New York University, New York, NY 10012.

\section{College Libraries Section (CLS)}

Vice-Chair/Chair-Elect: Janice C. Fennell, Director of Libraries, Georgia College, Milledgeville, GA 31061; Eleanor H. Pinkham, Director of Library and Media Services, Kalamazoo College, Kalamazoo, MI 49007.

Secretary: Larry Hardesty, Director of Library Services, Eckerd College, P.O. Box 12560, St. Petersburg, FL 33733; Daniel W. Lester, Director, John F. Reed Library, Fort Lewis College, Durango, CO 81301 .

\section{Community and Junior College Libraries Section (CJCLS)}

Vice-Chair/Chair-Elect: Billy C. Beal, Acquisitions Librarian, Meridian Junior College, Meridian, MS 39301; Shirley A. Lowman, Library Technical Services Director, Maricopa Community Colleges, 2325 E. McDowell Road, Phoenix, AZ 85006 .

Secretary: Louise Marsen, Media Specialist, Brookdale Community College LRC, Newman Springs Road, Lincroft, NJ 07738; Madison Mosley, Director, Learning Resources, South Florida Community College, $600 \mathrm{~W}$. College Dr., Avon Park, FL 33825.

\section{Education and Behavioral Sciences Section (EBSS)}

Vice-Chair/Chair-Elect: Inge Kauffman, Director of the Library, California School of Professional Psychology, 1350 M Street, Fresno, CA 93710; Nancy O'Brien, Associate Professor of Library Administration, University of Illinois Li- brary, Room 100, 1408 W. Gregory Dr., Urbana, IL 61801 .

Secretary: Julie Czisny, Falvey Library, Villanova University, Villanova, PA 19085; Tara Lynn Fulton, Assistant Interlibrary Loan/Reference Librarian, Northwestern University Library, Evanston, IL 60202.

\section{Law and Political Science Section (LPSS)}

Vice-Chair/Chair-Elect: Carole A. Larson, Social Sciences Reference Librarian, University of Nebraska at Omaha, Omaha, NE 68182-0237; Earl Shumaker, Head, Government Publications/ Microforms Department, University Libraries, Northern Illinois University, DeKalb, IL 60115.

Secretary: Susan Shiroma, Documents Librarian, Bobst Library, Social Science/Documents Center, New York University, 70 Washington Square South, New York, NY 10012; Christine E. Thompson, Head, Original Cataloging Department, Processing Division, Sterling C. Evans Library, Texas A\&M University, College Station, TX 77843-5000.

Member-at-Large: Sandra S. Coleman, Deputy Librarian, Harvard Law School, Cambridge, MA 02138; Eric M. Wedig, Government Documents Librarian, Memphis State University Libraries, Memphis, TN 38152.

\section{Rare Books and Manuscripts Section (RBMS)}

Vice-Chair/Chair-Elect: Ellen S. Dunlap, Director, Rosenbach Museum and Library, 2010 DeLancey Place, Philadelphia, PA 19103; William L. Joyce, Associate University Librarian for Rare Books and Special Collections, Princeton University Library, Princeton, NJ 08540.

Secretary: Lisa Browar, Curator of Rare Books and Manuscripts, Vassar College Library, Box 20, Poughkeepsie, NY 12601; Philip A. Metzger, Curator of Special Collections, Lehigh University, Linderman Library 30, Bethlehem, PA 18015-3067.

Member-at-Large: Dianne M. Chilmonczyk, Head, Special Collections, Catalog Section, Stanford University Libraries, Stanford, CA 94305; Cathy Henderson, Research Librarian, Harry Ransom Humanities Research Center, P.O. Box 7219, University of Texas, Austin, TX 78713-7219.

\section{Science and Technology Section (STS)}

Vice-Chair/Chair-Elect: Laura M. Osegueda, Agriculture and Life Sciences Librarian, D.H. Hill Library, Box 7111, North Carolina State University, Raleigh, NC 27695-7111; Susanne L. Redalje, Head, Chemistry-Pharmacy Library, 192 Bagley Hall, BG-10, University of Washington, Seattle, WA 98195 .

Secretary: Barbara A. Kautz, Entomology, Fisheries and Wildlife Librarian, 375 Hodson Hall, University of Minnesota, St. Paul, MN 55108; Dorothy McGarry, Head, Catalog Section, Physical Sciences and Technology Libraries, 8251 
Boelter Hall, University of California, Los Angeles. CA 90024.

\section{Slavic and East European Section (SEES)}

Vice-Chair/Chair-Elect: G. Koolemans Baynen, Slavic Cataloger and Reference Librarian, Ohio State University Libraries, 1858 Neil Avenue Mall, Columbus, OH 43210; Barbara A. Galik, Head, Slavic and East European Section, Suzzallo Library, FM-25, University of Washington, Seattle, WA 98103.

Secretary: Harold M. Leich, Slavic Acquisitions Librarian, Slavic and East European Library, University of Illinois, $1408 \mathrm{~W}$. Gregory Drive, Room 225, Urbana, IL 61801: Allan Urbanic, Librarian for Slavic Collections, General Library, Room 346. University of California. Berkeley, CA 94720 .

Member-at-Large: Donna A. Canevari de Paredes, Slavic Bibliographer. Collection Development, Main Library, University of Saskatchewan, Saskatoon, Saskatchewan S7N 0W0, Canada; Alexandra Filippenko, Slavic Librarian, University of California, Santa Barbara, CA 93106.
University Libraries Section (ULS)

Vice-Chair/Chair-Elect: Charles B. Osburn, Dean of Libraries, University of Alabama, Gorgas Library, P.O. Box S, Tuscaloosa, AL 35487-9784; Karen S. Seibert, Associate University Librarian for Public Services, Davis Library 080A, University of North Carolina, Chapel Hill, NC 27514.

\section{Western European Specialists Section (WESS)}

Vice-Chair/Chair-Elect: John Dillon, European Humanities Bibliographer, University of Wisconsin, Madison, WI 53706; Eva Sartori, Assistant Professor, Humanities/Social Sciences, University of Nebraska, Lincoln, NE 68588-0410.

Secretary: Ceres B. Birkhead, Foreign Languages Cataloger, Troy H. Middleton Library, Louisiana State University, Baton Rouge, LA 70803-3300; W. David Rozkuszka, Jonsson Library of Government Documents. Stanford University Libraries, Stanford. CA 94305.

Member-at-Large: John Bell Henneman Jr. History Bibliographer. Princeton University Library, Princeton, NJ 08544: Louis Pitschmann. Northern European Studies Librarian. Cornell University, Ithaca, NY 14853.

\section{ACRL meetings in Chicago}

\section{A tentative schedule for ALA's Midwinter Meeting, January 17-22, 1987.}

\section{ACRL BOARD OF DIRECTORS}

First meeting: Sunday, January 18, 2:00-5:30 p.m.

Second meeting: Tuesday, January 20, 2:00-5:30 p.m.

Orientation and luncheon: Sunday, January 18, 12:30-2:00 p.m.

Presidential Candidates Forum: Sunday. January 18, 8:00-10:00 p.m.

\section{ACRL DIVISIONAL COMMITTEES}

Academic Library Statistics: Saturday, January 17, 9:00-11:00 a.m.; Monday, January 19, 9:00-11:00 a.m.

Academic or Research Librarian of the Year Award: Monday, January 19, 2:00-4:00 p.m. *

* Meetings with an asterisk are closed meetings. 